\title{
Voluntary and reflex control of the biceps brachii muscle in spastic-athetotic patients
}

\author{
PETER D. NEILSON ${ }^{1}$ \\ From the Division of Neurology, the Prince Henry Hospital and the Schools \\ of Medicine and Physics, University of New South Wales, Sydney, Australia
}

SUMMARY A cross-correlation technique of analysis was used to measure the transmission characteristics of tonic stretch reflex (TSR) pathways in spastic-athetoid subjects sustaining a voluntary contraction in the biceps brachii muscle. A comparison was made with the transmission characteristics of normal subjects measured by the same technique. It was found that gain and phase characteristics of spastic patients did not display the large resonant peaks present in normals. It is proposed that the resonant peaks in the TSR transmission of normal subjects were caused by long loop pathways. The absence of these peaks in the spastic patients supports the hypothesis that short-circuiting of long loop pathways by hyperactive spinal reflexes is part of the mechanism of spasticity.

The tonic vibration reflex (TVR) has been shown by Matthews (1966) to depend on excitation of primary muscle spindle endings. Investigations of the TVR in man indicate that the reflexes observed in spasticity may not simply be exaggerated normal tonic reflexes (De Gail, Lance, and Neilson, 1966; Hagbarth and Eklund, 1968). In normal man the TVR is slowly augmenting, whereas in spastic man vibration causes a rapid onset of muscle contraction similar to that recorded in the decerebrate cat (Gillies, Burke, and Lance, 1971). It is proposed that hyperactive spinal reflexes may shortcircuit long loop pathways as a part of the mechanism of spasticity. If this hypothesis is correct, the tonic stretch reflex (TSR) transmission characteristics measured in spastic patients should be different from those of normal subjects.

In patients in whom the TSR is hyperactive an electromyographic (EMG) response to muscle stretch can be elicited by passive displacement of the limb. In relaxed normal subjects, however, the limb can be moved passively without encountering any active resistance from the muscle. To obtain a response to passive stretching the reflexes must be activated deliberately. Voluntary contraction of the muscle under test

1 Centre Industries Research Scholar. activates the TSR of that muscle in normal subjects (Hammond, 1955; Dewhurst, 1967) but the EMG responses are difficult to measure separately because they are mixed with muscle potentials due to the voluntary contraction.

A cross-correlation technique of analysis has been described (Neilson, 1972b) which separates the EMG reflex responses from the total electromyographic activity. Using this technique, it was found that the transmission characteristics of TSR pathways activated by voluntary contraction in normal subjects were more complex than previously described for decerebrate cats (Poppele and Terzuolo, 1968; Rosenthal, McKean, Roberts, and Terzuolo, 1970) or spinal man (Burke, Andrews, and Gillies, 1971). It was suggested that the peaks of resonance in the gain and phase characteristics might be caused by interactions with long loop reflex pathways which could involve brain-stem, cerebellum, basal ganglia, and sensorimotor cortex.

The aim of this study is to test the hypothesis that short-circuiting of long loop pathways is a part of the mechanism of spasticity. By using the cross-correlation technique of analysis it is possible to measure the TSR transmission characteristics of the biceps brachii muscle in spastic-athetoid patients while they sustain a voluntary contraction in the muscle being tested. 
The characteristics obtained can thus be compared and contrasted with those measured previously in normal subjects using the same technique (Neilson, 1972b).

\section{METHOD}

Ten cerebral palsied (CP) quadriparetic patients were selected for study because the clinical picture was predominantly one of spasticity and no athetotic movement was apparent at rest.

The characteristics for both voluntary and reflex control of biceps brachii muscle were measured using the free wheel test, kinaesthetic tracking test, and limb stabilization test described previously (Neilson, 1972a; 1972b). The range, speed, power, and accuracy of voluntary arm movement was assessed for each patient. Subjects lay supine on a couch with the right arm strapped into a frame which constrained movement to flexion-extension about the elbow. Goniometers attached to both arms allowed elbow angles to be recorded on a Grass polygraph. Beckman EMG surface electrodes attached $5 \mathrm{~cm}$ apart over the biceps muscle of the right arm were used to record direct EMG and integrated EMG (IEMG).

In the free-wheel test patients were asked to oscillate the right arm rhythmically about the $90^{\circ}$ position as rapidly as possible. For the kinaesthetic tracking test the left arm was moved randomly by the experimenter. The subject was required to move the right arm voluntarily so as to keep left and right elbow angles aligned to similar positions. Angular positions of both elbow joints had to be perceived by the kinaesthetic sensory system so that the right arm position could be adjusted to nullify the alignment error. Because of the possible criticism that the tracking performance may have been limited by the patient's inability to perceive joint position or by associated movements of the right arm caused by passive displacement of the left, voluntary control of right arm position was also assessed on all subjects by a visual tracking test. The goniometer attached to the right arm controlled the vertical position of a horizontal line on an oscilloscope screen. A second, not so bright and slightly defocused line on the screen was randomly driven up and down by the experimenter. The patient's task was to keep the two lines superimposed by adjusting the position of the right elbow angle.

For the limb stabilization test the patient was instructed to hold the right arm as still as possible in the $90^{\circ}$ position in spite of disturbance torques about the elbow applied by a stretching machine connected to the arm frame by a spring. Elbow angle changes were used as a measure of changes in length of biceps muscle and reflex responses were recorded as EMG and IEMG signals.

\section{TECHNIQUES OF ANALYSIS}

'Alpha-gamma linkage' emphasizes that voluntary movement is controlled by a coordinated action of $\bar{\omega}$ both alpha and gamma motoneurones (Granit, 1966; To Granit and Kellerth, 1967). It has been proposed that stretch reflexes might act as a position follow-up servosystem to contract extrafusal muscle fibres (Merton, 1953). This servo model could work equally well if the stretch reflexes were mediated by long loop pathways in addition to the monosynaptic arc. In- $\overline{0}$ deed, it has been suggested that the sensitivity of the $\frac{\bar{\sigma}}{\sigma}$ monosynaptic reflex arc alone may not be sufficient for an effective servo action (Matthews, 1966). Since the TSR has been regarded as a servo control system, it seems reasonable to borrow a technique of analysis os from control theory to measure the open loop trans- $\vec{O}$ fer function of the neurological TSR pathways. Transfer function is a concept which has been well $\omega$ developed in control theory providing a mathematical description of the input-output relationship of a system. The transfer function of a linear system can $\omega^{\prime}$ be represented graphically by frequency response or curves which consist of gain and phase plotted of against frequency. Frequency response curves hate the advantage that they completely specify the sy్ㅠ 윽 tem; consequently, any changes in reflex transmission should be reflected by changes in the curves.

EMG was used as a measure of neuronal outpgt $\frac{\bigcirc}{0}$ from TSR pathways. This signal was expected $\$$ contain muscle potentials due to both voluntary an reflex activity. It was anticipated that voluntajy activity would not be correlated with muscle stretch-? ing and so could be thought of as noise contaminating the reflex signal. Harmonic distortion due to non-linear TSR transmission could also increase the $\bar{\partial}$ amount of EMG activity not correlated with muscle stretching. Because of this contaminating noise $a \frac{\Phi}{\mathbb{2}}$ statistical method of analysis was used to average $\overrightarrow{\vec{H}}$

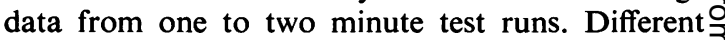
frequencies of stretching were used on each run and correlation functions and power spectra were calculated using methods described by Jenkins and Watts (1968). Spectral composition of the signals was thus measured so that harmonic distortion could be de-? tected and reflex responses could be separated from the total EMG activity.

The relationship between IEMG of right biceps $\frac{\mathrm{O}}{3}$ muscle and change of right elbow angle, recorded during the free-wheeling test, was determined using correlation and spectral analysis. The relationship provided a description of the input-output charac-三. teristics of contractile mechanisms in series with N muscle and limb mechanics. 


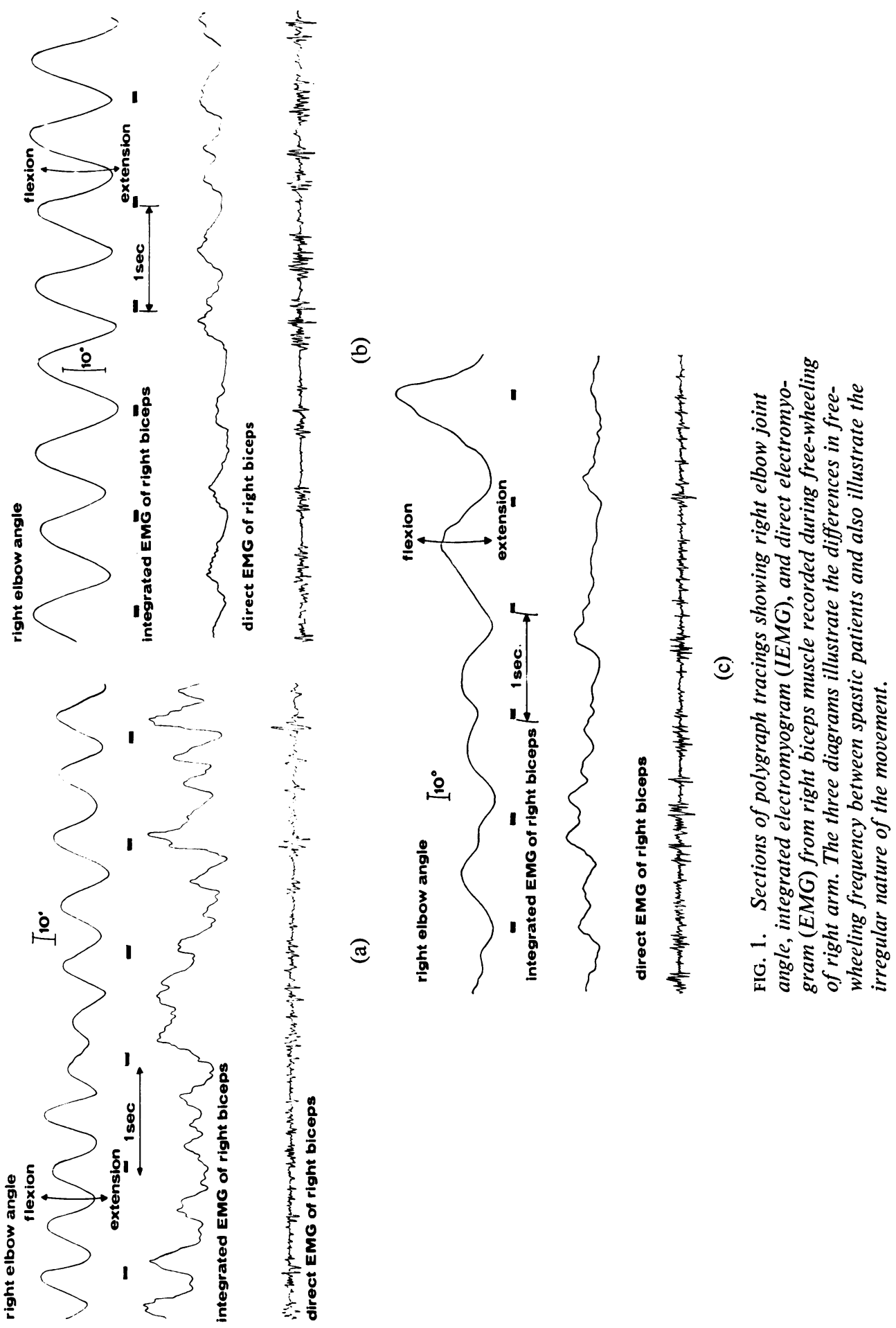


The same technique was used to determine the transfer characteristics between the passively moved left elbow angle and the voluntarily positioned right elbow angle measured during the kinaesthetic tracking test.

Transmission characteristics of the neurological pathways concerned with the TSR of voluntarily contracted biceps muscle were measured during the limb stabilization test. Spectral analysis was used to calculate the frequency response curves relating right elbow angle changes to the IEMG reflex responses from the right biceps muscle.

\section{RESULTS}

FREE-WHEELING When spastic-athetoid patients were asked to oscillate the right arm back and forth as rapidly as possible the frequency of movement varied from subject to subject up to a maximum of $2.0 \mathrm{~Hz}$ (Fig. 1). Simultaneous recording of EMG from biceps and triceps muscles showed that in three of the 10 patients activity in the two muscles alternated and the antagonistic muscle was completely inactive as in normal subjects while in the remainder EMG persisted in the antagonistic muscle. The power spectrum of elbow angle signal (Fig. 2) recorded during free-wheeling contained a large peak, indicating that for all patients the movement was predominantly at one frequency within the range $0 \cdot 6-2 \cdot 0 \mathrm{~Hz}($ mean $=1 \cdot 2 \mathrm{~Hz})$.

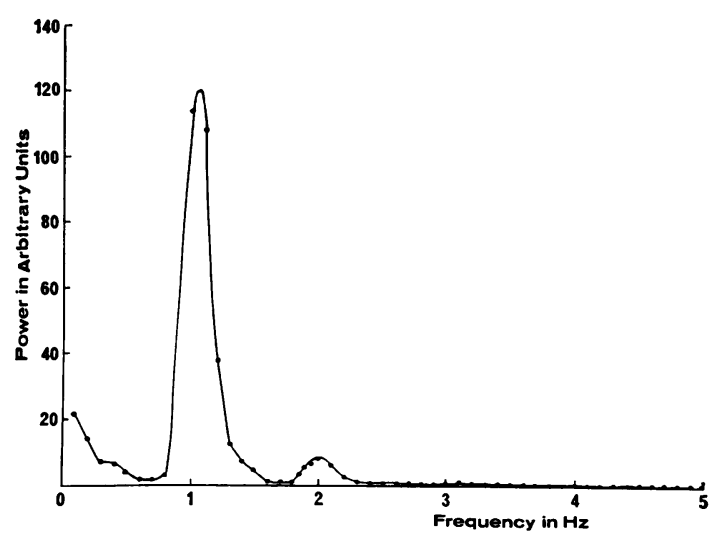

FIG. 2. An auto-power spectrum of a right elbow joint angle signal recorded from a spastic patient freewheeling the right arm. The large peak indicates that the movement was predominantly at a frequency of $1 \cdot 0 \mathrm{~Hz}$.
After correcting for the phase lag in the IEMG signal introduced by the integrating filter $(T=$ $0.16 \mathrm{sec}$ ), the cross-power spectra between IEMG

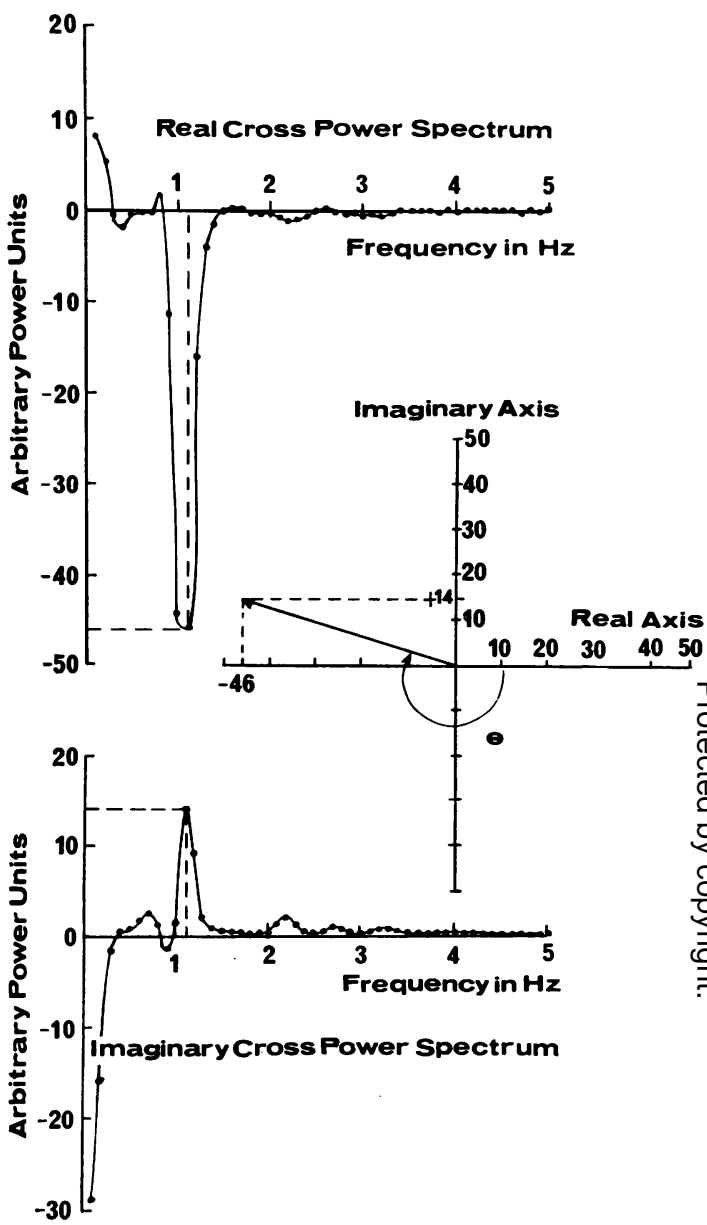

FIG. 3. (Top) A graph showing the real component of the cross-power spectrum between IEMG from right biceps muscle and right elbow angle changes recorded from a spastic subject free-wheeling the right arm. (Bottom) Agraph showing the imaginary or quadrature component of the same cross-power spectrum. (Middle) An argand diagram illustrating the phase lag of joint angle flexion movements behind IEMG activity of biceps muscle at the predominant freewheeling frequency. The values of the real and imaginary components plotted were measured from the top and bottom graphs respectively. The phase lag has to be increased by $45^{\circ}$ to correct for the phase lag introduced into the IEMG signal by the integrating filter. 


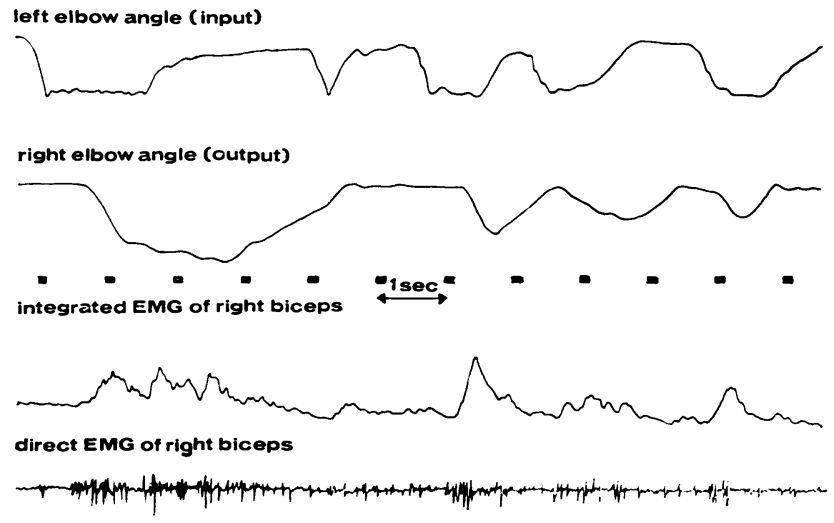

(a)

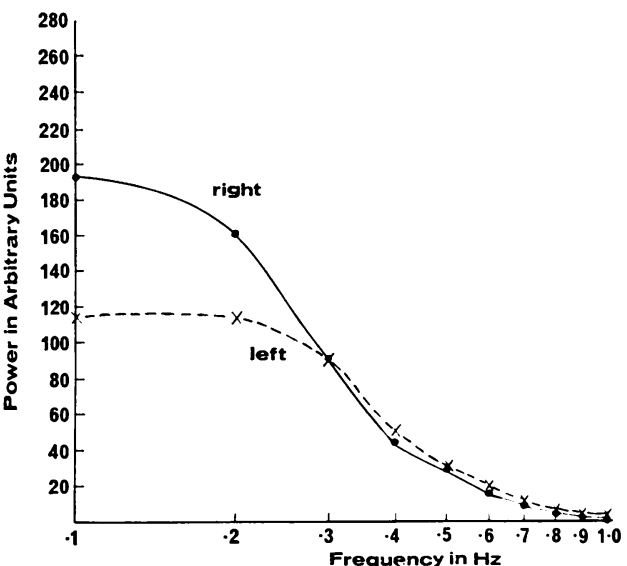

(b)

FIG. 4. (a) Section of polygraph tracing of left elbow angle, right elbow angle, integrated EMG, and direct EMG of right biceps brachii muscle of a spastic patient recorded during a kinaesthetic tracking test. (b) Power spectra of left and right elbow angle signals recorded from a spastic patient during a kinaesthetic tracking test. Passive movements of the left arm were not permitted to exceed frequencies of about $1.5 \mathrm{~Hz}$ because the patients could not track at these high speeds.

and elbow angle changes indicated a $180^{\circ}-270^{\circ}$ phase lag of elbow flexion movement behind EMG activity of the biceps muscle (Fig. 3). This large phase lag was present in nine out of 10 patients tested. The remaining patient had a low free wheeling frequency $(0.6 \mathrm{~Hz})$ and had only a $6^{\circ}$ phase lag of elbow flexion behind EMG of biceps.

KINAESTHETIC TRACKING In the kinaesthetic tracking tests patients were unable to track at high speed and therefore the power spectrum of the passively moved left arm was not permitted to exceed $1.5 \mathrm{~Hz}$ (Fig. 4). Tracking performance was generally very poor as indicated by the coherence functions (Fig. 5). All patients had low coherence between left and right elbow angles at frequencies of less than $0.3 \mathrm{~Hz}$. The maximum value of the coherence was always less than 0.5 and for all patients it diminished to zero by $1.0 \mathrm{~Hz}$.

The power spectrum of the misalignment or error between left and right elbow angles (Fig. 6) did not contain a peak at frequencies where the coherence was decreasing toward zero as previously found in normal subjects (Neilson, 1972a).
Amplitude and phase frequency response curves describing the relationship between the coherent components of left and right arm move-

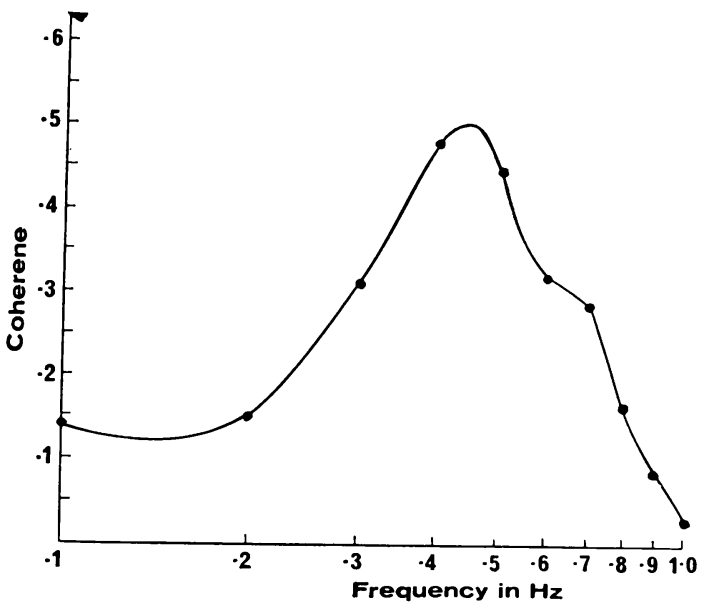

FIG. 5. A graph indicating the coherence between left and right elbow angle signals recorded from a spastic patient during a kinaesthetic tracking test. The maximum value of 0.5 was the largest value of coherence measured in all of the ten patients. The coherence functions all decrease to zero by a frequency of $1.0 \mathrm{~Hz}$. 


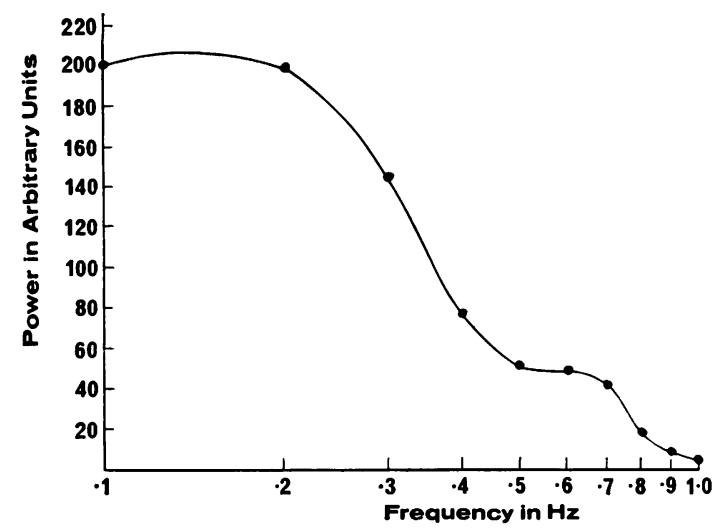

FIG. 6. A power spectrum of the error between left and right elbow angle signals recorded from a spastic patient during a kinaesthetic tracking test.

ments emphasized the differences in performance between subjects as illustrated in Fig. 7 .

Results of the visual tracking tests were similar to those of kinaesthetic tracking. Thus poor performance was not due to associated involuntary movements of the right limb evoked by passive displacement of the opposite arm.

LIMB STABILIZATION The EMG responses from biceps brachii muscle to stretch were coherent with joint angle changes up to $10.0 \mathrm{~Hz}$, the highest frequency tested (Fig. 8). As in the previous tests, the frequency response curves showed differences between spastic patients. In spite of these differences the TSR transmission characteristics of all the spastic patients demonstrated important differences from those measured previously in normal subjects. The gain curves did not display large peaks of resonance and the phase lead of EMG ahead of muscle stretch was less than in normal subjects. Gain and phase frequency response curves from three spastic patients have been graphed (Fig. 9) and contrasted with those of normal man reported previously (Neilson, 1972b).

\section{DISCUSSION}

FREEWHEELING The fastest voluntary movement $\stackrel{\omega}{\rho}$ of the arm was $0.6-2.0 \mathrm{~Hz}($ mean $=1.2 \mathrm{~Hz})$ in $\overline{3}$ spastic patients compared with $4 \cdot 0-6 \cdot 0 \mathrm{~Hz}$ in normal subjects under similar test conditions. of Elbow flexion movements lagged $180^{\circ}-270^{\circ}$ iw phase behind EMG activity in the biceps muscle suggesting that the maximum speed of movement 은 was limited by the mechanical load on the com tractile elements of the muscle. This can be cor

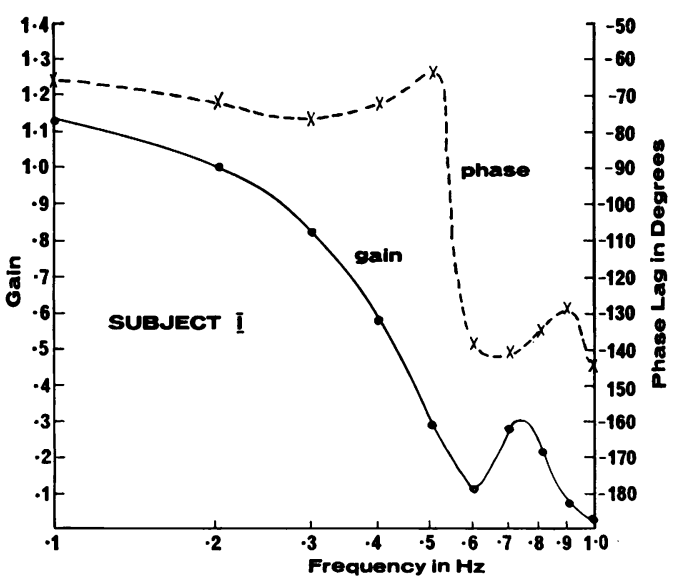

(a)

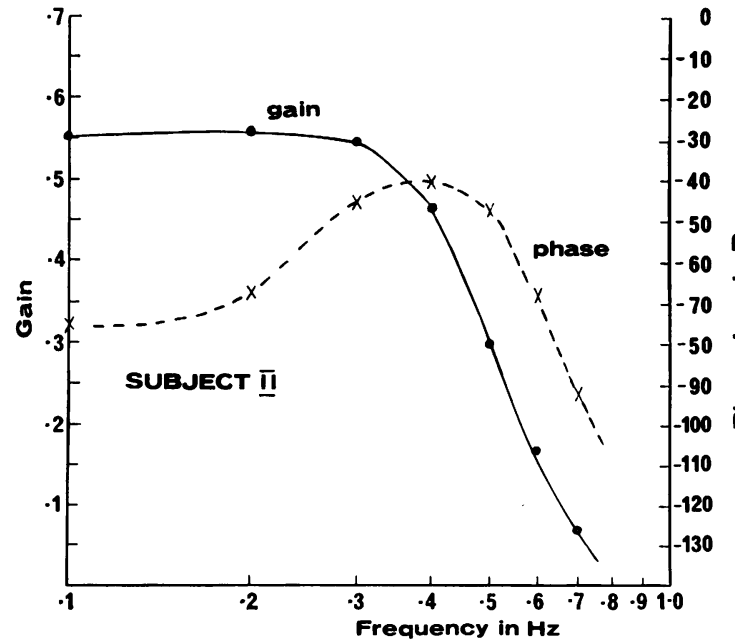

(b)

FIG. 7. Amplitude and phase frequency response characteristics describing the relationship between left and $\frac{\mathrm{D}}{\mathrm{O}}$ right elbow angle signals recorded from spastic patients during kinaesthetic tracking tests. The two curves are from two different patients indicating the differences in tracking performance. 


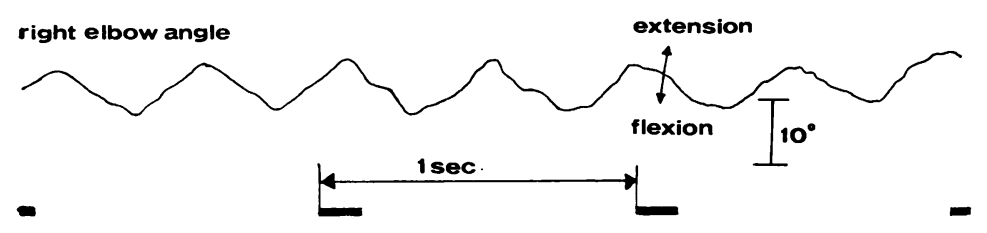

integrated EMG

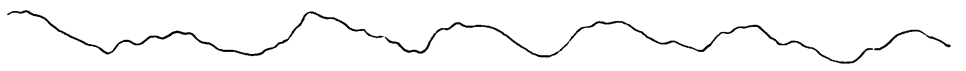

direct EMG

FIG. 8. Section of a polygraph tracing showing right elbow angle changes, IEMG and EMG signals from right biceps muscle recorded from a spastic patient during a limb stabilization test. IEMG responses were coherent with joint angle changes up to 10.0 $\mathrm{Hz}$, the highest frequency

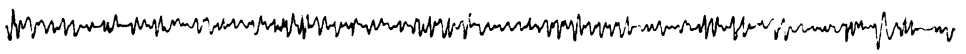
tested.

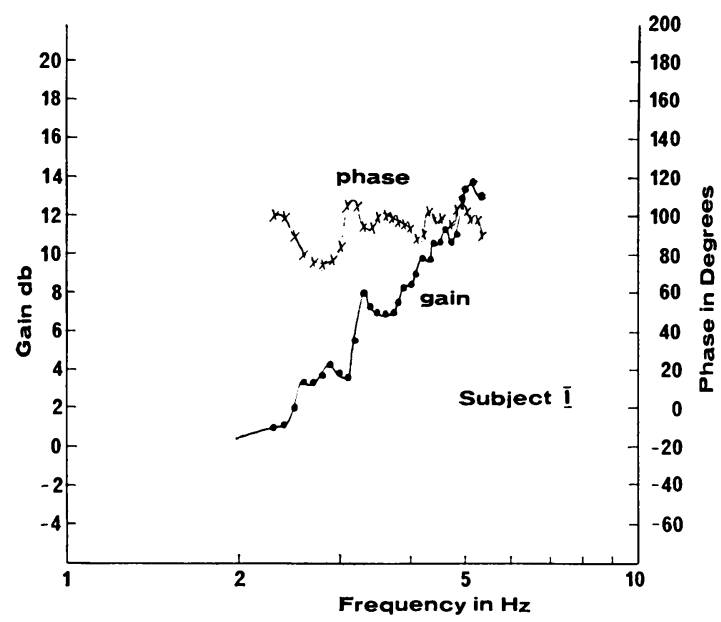

(a)

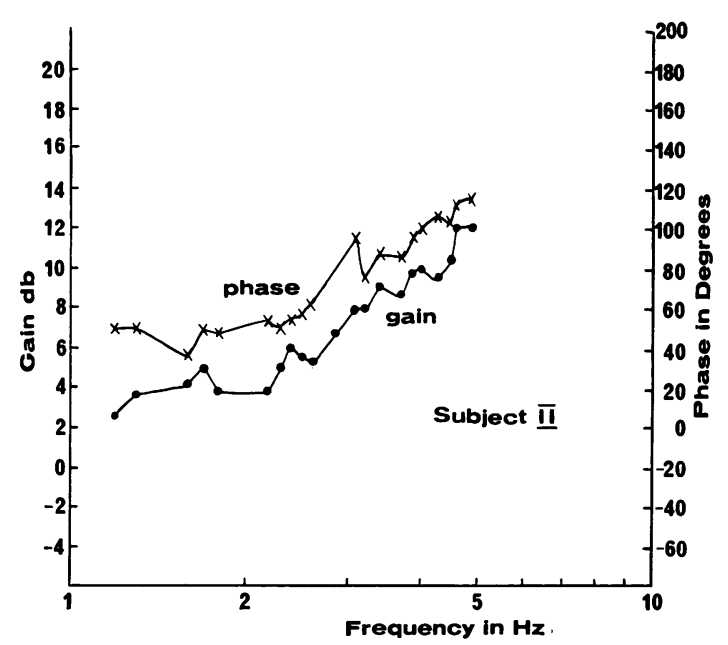

(b)

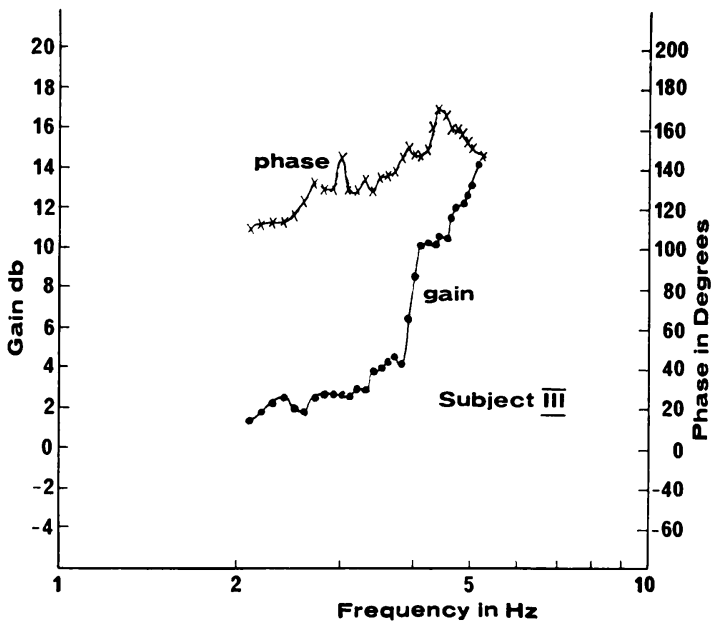

(c)

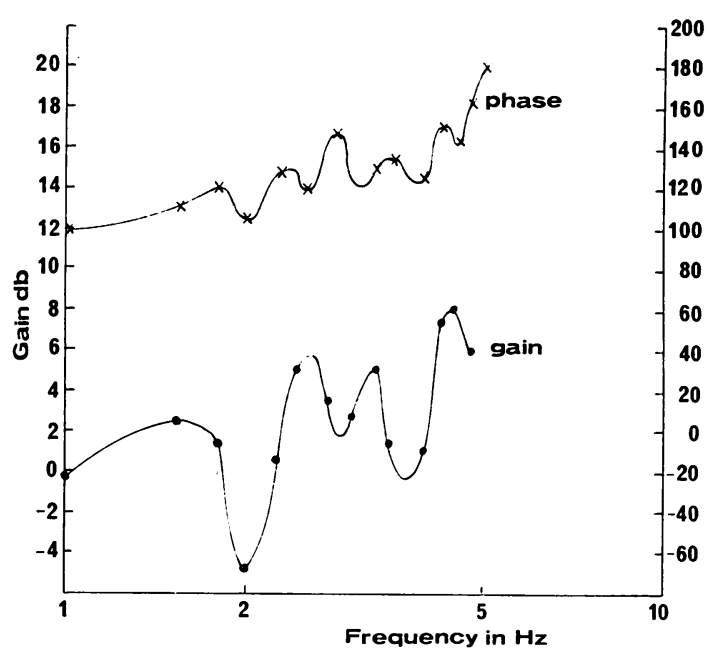

(d)

FIG. 9. Gain and phase frequency response curves describing TSR transmission in three spastic patients, (a), (b), (c), have been contrasted with the curves from a normal subject (d). The gain curves of the spastic patients do not display resonant peaks. 
cluded by analogy with a pendulum, the speed of movement of which is limited by the mechanical property of inertia. At high speed the pendulum displacement lags $180^{\circ}$ in phase behind the displacing force. Similarly, at speeds of movement limited by limb inertia one would expect joint angle changes to lag by $180^{\circ}$ in phase behind muscle tension.

The total mechanical load on the contractile elements of muscle is comprised of muscle viscoelastic elements, limb inertia, gravitational forces, and torques developed about the joints by antagonistic muscles. Voluntary flexion about the elbow could be opposed by hypersensitive stretch reflexes in triceps muscle and so the abnormal mechanical load on the biceps muscle may be a consequence of the inability of central mechanisms to inhibit the TSR of antagonistic muscles. However in the three patients in whom the EMG activity of the antagonistic muscle was completely suppressed, the large phase lag was still measured. Therefore, some other mechanism must be invoked to explain the increase in mechanical load on the biceps muscle. Perhaps morphological changes in muscle structure (which may occur within days if a patient maintains a fixed posture) may increase the mechanical load. Whatever the cause, the large phase lag measured at the low free-wheeling frequency emphasizes that the relationship between neural input and shortening of biceps brachii muscle in spastic-athetotic patients differs from that of normal subjects.

KINAESTHETIC TRACKING Tracking by spastic patients generally was very poor. Even at frequencies as low as $0.3 \mathrm{~Hz}$ more than $50 \%$ of right arm movements made during the test were not correlated with passive movements of the left arm. This poor correlation was indicated by the low value $(<0.5)$ of the coherence function. The incoherent movements could perhaps best be described as inappropriate voluntary movements. Although the cerebral palsied patients presented a clinical picture of spasticity with no athetotic movement at rest, the extra sensitivity of this test may have revealed an associated athetosis responsible for the inappropriate movements.

High speed tracking was not limited by a loss of stimulus-response synchronization as it was with normals, but by a dropping off in the amplitude of the response. The absence of a peak in the error power spectrum shows the absence of high speed incoherent movements similar to those demonstrated in normal subjects when endeavouring to track a signal at high frequency. For all patients tested the coherence function had decreased to zero before reaching a frequency of $1.0 \mathrm{~Hz}$.

Limb STABILIZATION During the limb stabilization test IEMG responses were coherent with joint angle changes up to $10.0 \mathrm{~Hz}$, the highest frequency tested. For frequencies greater than $1.0 \mathrm{~Hz}$ at least, these EMG responses must have been caused by involuntary or reflex systems, since it has been shown above that the patients could not produce a coherent voluntary response at these frequencies.

Sensitivity of the TSR in spastic patients was greater than measured in normals but this has not been emphasized in the graphs, since they have been normalized to $0 \mathrm{db}$ at $1.0 \mathrm{~Hz}$.

No large peaks of resonance were measured in the TSR transmission characteristics of spastico patients. This is a clear and striking differences from the TSR characteristics of normal subjects 0 described previously. The gain curves of allo $\frac{f}{0}$ normal subjects displayed sharply tuned peaks? Such complex reflex transmission cannot be exto plained by known spinal pathways. It has been argued that the resonant peaks are caused by interactions between long loop paths, which are activated by voluntary contraction. This argument is supported by a number of experimental results. Spinobulbospinal reflexes have been investigated, confirming the existence of long loop pathways from cutaneous, joint, and muscle afferent nerve fibres in the cat, dog, monkey, and in man (Shimamura and Livingston, 1963; Shimamura, Mori, Matsushima, and Fujimori, 1964; Shimamura and Akert, 1965). Long latency $(80 \mathrm{msec})$ EMG responses of tibial muscle to percutaneous stimulation of the tibial nerve in man was thought to be an example of a long loop reflex response (Shimamura et al., 1964). The magnitude of the response could be greatly augmented by voluntary dorsiflexion of the foot. This augmentation is consistent with the proposition that long loop reflexes are activated by voluntary contraction. H-reflex recovery cycles 
have been shown to exhibit a long latency (50$100 \mathrm{msec}$ ) facilitation which cannot be explained by spinal processes. Long loop pathways have been proposed to explain this phenomenon (Taborikova, Provini, and Decandia, 1966; Yap, 1967). There is ample evidence that pathways from muscle afferent fibres project to brain-stem, cerebellum, thalamus, and sensorimotor cortex (Matthews, Philips, and Rushworth, 1957; Lundberg and Winsbury, 1960; Oscarsson and Rosen, 1963; Andersson, Landgren, and Wolsk, 1966; Oscarsson, Rosen, and Sulg, 1966; Jansen, Poppele, and Terzuolo, 1967; Narabayashi, Goto, and Kubota, 1968) and also that synaptic transmission in afferent pathways can be influenced by supraspinal centres (Kuno and Perl, 1960; Higgins, Partridge, and Glaser, 1962; Lundberg, 1964; Magladery, 1964; Lundberg, 1967). It therefore seems plausible that when a man voluntarily contracts a muscle, basal ganglia, brain-stem, and spinal centres might be activated which in turn activate long loop reflex pathways.

Absence of large resonant peaks in the TSR characteristics of spastic patients indicates that the reflexes of spasticity are not merely exaggerated normal stretch reflexes. The evidence from this study supports the hypothesis that exaggerated sensitivity of spinal reflexes in spastic patients causes them to dominate or short circuit long loop pathways.

I wish sincerely to thank Associate Professor J. W. Lance and Professor E. P. George for their valued advice and encouragement and for their generous provision of equipment from the Schools of Medicine and Physics. I also thank Miss Susan Casey who drew the Figures. I gratefully acknowledge the Spastic Centre of N.S.W. for the Centre Industries Research Scholarship which has provided the financial support for this project.

\section{REFERENCES}

Andersson, S. A., Landgren, S., and Wolsk, D. (1966). The thalamic relay and cortical projection of group I muscle afferents from the forelimb of the cat. Journal of Physiology, 183, 576-591.

Burke, D., Andrews, C. J., and Gillies, J. D. (1971). The reflex response to sinusoidal stretching in spastic man. Brain. 94, 455-470.

De Gail, P., Lance, J. W., and Neilson, P. D. (1966). Differential effects on tonic and phasic reflex mechanisms produced by vibration of muscles in man. Journal of Neurology, Neurosurgery, and Psychiatry, 29, 1-11.
Dewhurst, D. J. (1967). Neuromuscular control system. IEEE Transactions of Bio-Medical Engineering, 14, 167-171.

Evarts, E. V. (1968). Relation of pyramidal tract activity to force exerted during voluntary movement. Journal of Neurophysiology, 31, 14-27.

Gillies, J. D., Burke, D. J., and Lance, J. W. (1971). Tonic vibration reflex in the cat. Journal of Neurophysiology, 34, 252-262.

Granit, R. (1966). Effects of stretch and contraction on the membrane of motoneurones. In Muscular Afferents and Motor Control. Proceedings of the 1st Nobel Symposium, 1965, Södergarn, pp. 37-50. Edited by R. Granit. Almqvist and Wiksell: Stockholm.

Granit, R., and Kellerth, J.-O. (1967). The effects of stretch receptors on motoneurones. In Neurological Basis of Normal and Abnormal Motor Activities, pp. 3-28. Edited by M. D. Yahr and D. P. Purpura. Raven Press: New York.

Grillner, S., Hongo, T., and Lund, S. (1969). Descending monosynaptic and reflex control of $\gamma$-motoneurones. Acta Physiologica Scandinavica, 75, 592-613.

Hagbarth, K.-E., and Eklund, G. (1968). The effects of muscle vibration in spasticity, rigidity, and cerebellar disorders. Journal of Neurology, Neurosurgery, and Psychiatry, 31, 207-213.

Hammond, P. H. (1955). Involuntary activity in biceps following the sudden application of velocity to the abducted forearm. Journal of Physiology, 127, 23-25.

Higgins, D. C., Partridge, L. D., and Glaser, G. H. (1962). A transient cerebellar influence on stretch responses. Journal of Neurophysiology, 25, 684-692.

Jansen, J. K. S., Poppele, R. E., and Terzuolo, C. A. (1967). Transmission of proprioceptive information via the dorsal spinocerebellar tract. Brain Research, 6, 382-384.

Jenkins, G. M., and Watts, D. G. (1968). Spectral Analysis and its Applications. Holden-Day: San Francisco.

Kuno, M., and Perl, E. R. (1960). Alteration of spinal reflexes by interaction with suprasegmental and dorsal root activity. Journal of Physiology, 151, 103-122.

Lundberg, A. (1964). Supraspinal control of transmission in reflex paths to motoneurones and primary afferents. Progress in Brain Research, 12, 197-221.

Lundberg, A. (1967). The supraspinal control of transmission in spinal reflex pathways. Electroencephalography and Clinical Neurophysiology, Suppl. 25, 35-46.

Lundberg, A., and Winsbury, G. (1960). Functional organization of the dorsal spino-cerebellar tract in the cat. VI. Acta Physiologica Scandinavica, 49, 165-170.

Magladery, J. W. (1964). Central facilitating and inhibiting mechanisms in the control of muscle tone. Clinical and Pharmacological Therapeutics, 5, 805-811.

Matthews, P. B. C. (1966). The reflex excitation of the soleus muscle of the decerebrate cat caused by vibration applied to its tendon. Journal of Physiology, 184, 450-472.

Matthews, P. B. C., Philips, C. G., and Rushworth, G. (1958). Afferent systems converging upon cerebellar Purkinje cells in the frog. Quarterly Journal of Experimental Physiology, 48, 38-52.

Merton, P. A. (1953). Speculations on the servo-control of movement. In The Spinal Cord: a CIBA Foundation Symposium, pp. 247-260. Edited by G. E. W. Wolstenholme. Churchill: London.

Narabayashi, H., Goto, A., and Kubota, K. (1968). Projection of muscle afferents to the human thalamus. Proceedings of the Australian Association of Neurology, 5, 25-26.

Neilson, P. D. (1972a). Speed of response or bandwidth of voluntary system controlling elbow position in intact man. Medical and Biological Engineering, 10, 450-459. 
Neilson, P. D. (1972b). Frequency response characteristics of the tonic stretch reflexes of biceps brachii muscle in intact man. Medical and Biological Engineering, 10, 460-472.

Oscarsson, O., and Rosén, I. (1963). Projection to cerebral cortex of large muscle-spindle afferents in forelimb nerves of the cat. Journal of Physiology, 169, 924-945.

Oscarsson, O., Rosén, I., and Sulg, I. (1966). Organization of neurones in the cat cerebral cortex that are influenced from Group I muscle afferents. Journal of Physiology, 183, 189210.

Poppele, R. E., and Terzuolo, C. A. (1968). Myotatic reflex: its input-output relation. Science, 159, 743-745.

Rosenthal, N. P., McKean, T. A., Roberts, W. J., and Terzuolo, C. A. (1970). Frequency analysis of stretch reflex and its main subsystems in triceps surae muscles of the cat. Journal of Neurophysiology, 33, 713-749.
Shimamura, M., and Akert, K. (1965). Peripheral nervous relations of propriospinal and spino-bulbo-spinal reflex system. Japanese Journal of Physiology, 15, 638-647.

Shimamura, M., and Livingston, R. B. (1963). Longitudinal conduction systems serving spinal and brain-stem coordination. Journal of Neurophysiology, 26, 258-272.

Shimamura, M., Mori, S., Matsushima, S., and Fujimori, B. (1964). On the spino-bulbo-spinal reflex in dogs, monkeys and man. Japanese Journal of Physiology, 14, 411-421.

Taborikova, H., Provini, L., and Decandia, M. (1966). Evidence that muscle stretch evokes long-loop reflexes from higher centres. Brain Research, 2, 192-194.

Yap, C.-B. (1967). Spinal segmental and long-loop reflexes on spinal motoneurone excitability in spasticity and rigidity. Brain, 90, 887-896. 